\title{
THE VIRIAL THEOREM AND ITS APPLICATION TO THE SPECTRAL THEORY OF SCHRÖDINGER OPERATORS
}

\author{
BY JOACHIM WEIDMANN \\ Communicated by Felix Browder, January 4, 1967
}

1. Introduction. Let us consider elliptic differential operators of the form

$$
H=-\Delta+q(x), \quad x \in R^{m},
$$

where the potential $q(x)$ satisfies the following conditions:

(I) $q \in Q_{\alpha}\left(R^{m}\right)$ for some $\alpha>0$; i.e.

$$
M_{q}(x)=\int_{|x-y| \leqq 1}|q(y)||x-y|^{4-m-\alpha} d y
$$

is uniformly bounded for $x \in R^{m}$.

(II) For every $x \in R^{m}, x \neq 0$, there exists a radial derivative $q_{r}(x)$ of $q(x)$ and

$$
\epsilon^{-1}|q((1+\epsilon) x)-q(x)| \leqq q_{0}(x) \in Q_{\beta}\left(\boldsymbol{R}^{m}\right)
$$

holds for $0<\epsilon<\epsilon_{0}$ and some $\beta>0$; in particular we have $r q_{r}(x) \leqq q_{0}(x)$; hence $r q_{r} \in Q_{\beta}\left(R^{m}\right)$.

Under these conditions we shall prove in $\$ 2$ a very general form of the Virial Theorem of quantum mechanics. In $\S \S 3$ and 4 this theorem will be used to deduce some results on the spectrum of $H$.

Let $L_{2}\left(R^{m}\right)$ be the Hilbert space of functions which are squaresummable over $R^{m}$; the inner product in this space will be denoted by $\langle\cdot, \cdot\rangle$, the norm by $|\cdot|$.

From condition (I) one can conclude (e.g. Ikebe-Kato [2]):

(1) The operator $H$ with domain $D(H)=H_{2}\left(R^{m}\right)$ is selfadjoint in $L_{2}\left(R^{m}\right)\left(H_{2}\left(R^{m}\right)\right.$ is the closure of $C_{0}^{\infty}\left(\boldsymbol{R}^{m}\right)$ with respect to the norm $\left.|u|_{2}=\left\{\sum_{j, k}\left|\partial^{2} u /\left(\partial x_{j} \partial x_{k}\right)\right|^{2}+\sum_{j}\left|\partial u / \partial x_{j}\right|^{2}+|u|^{2}\right\}^{1 / 2}\right)$.

(2) For $u \in D(H)$ and $q \in Q_{\alpha}\left(R^{m}\right)$ we have $q u \in L_{2}\left(R^{m}\right)$.

(3) For $u, v \in D(H)$ we have $\Delta u, \Delta v \in L_{2}\left(R^{m}\right)$ and $\langle\Delta u, v\rangle=\langle u, \Delta v\rangle$.

\section{The Virial Theorem.}

THEOREM. Let conditions (I) and (II) be satisfied. If $\lambda$ is an eigenvalue of $H, u(x)$ a corresponding eigenfunction, then

$$
\left\langle\left(2 q+r q_{r}-2 \lambda\right) u, u\right\rangle=0, \quad 2\langle-\Delta u, u\rangle=\left\langle r q_{r} u, u\right\rangle .
$$


REMARK. The second equation is known in quantum mechanics as the virial theorem. (No proof seems to be known for this general case.)

Proof of the Theorem. Without any restriction we may assume that $u(x)$ is real-valued. Since $u(x)$ is an eigenfunction corresponding to the eigenvalue $\lambda$, it follows that

$$
\begin{aligned}
-\Delta u(x)+q(x) u(x) & =\lambda u(x), \\
-\Delta u(a x)+a^{2} q(a x) u(a x) & =a^{2} \lambda u(a x) .
\end{aligned}
$$

Every single term is in $L_{2}\left(R^{m}\right)$. Multiplication of the first equation by $u(a x)$, the second equation by $u(x)$ and integration over $R^{m}$ yields

$$
\begin{aligned}
(1 & \left.-a^{2}\right) \lambda \int_{R^{m}} u(x) u(a x) d x \\
& =\int_{R^{m}}\left\{-u(a x) \Delta u(x)+u(x) \Delta u(a x)+\left(q(x)-a^{2} q(a x)\right) u(x) u(a x)\right\} d x .
\end{aligned}
$$

Since $u(x)$ and $u(a x)$ are elements of $H_{2}\left(R^{m}\right)=D(H)$ it follows from (3) that, for $a>0$,

$$
F(a)=\int_{R^{m}}\left\{\left(1-a^{2}\right) \lambda+a^{2} q(a x)-q(x)\right\} u(x) u(a x) d x=0 .
$$

Consequently for any $\epsilon>0$ we have

$$
\begin{aligned}
& \epsilon^{-1} F(1+\epsilon) \\
& =\epsilon^{-1} \int_{R^{m}}\left\{-\left(2 \epsilon+\epsilon^{2}\right) \lambda+\left(1+2 \epsilon+\epsilon^{2}\right) q((1+\epsilon) x)\right. \\
& -q(x)\} u(x) u((1+\epsilon) x) d x \\
& =\int_{R^{m}}\left\{-(2+\epsilon) \lambda+\epsilon^{-1}(q((1+\epsilon) x)-q(x))\right. \\
& \quad+2 q((1+\epsilon) x)+\epsilon q((1+\epsilon) x)\} u(x) u((1+\epsilon) x) d x=0 ;
\end{aligned}
$$

hence the limit for $\epsilon \rightarrow 0$ must also vanish.

If we are able to show that this limit can be taken under the integral sign, we have

$$
\int_{R^{m}}\left\{-2 \lambda+r q_{r}+2 q\right\} u^{2}(x) d x=\left\langle\left(2 q+r q_{r}-2 \lambda\right) u, u\right\rangle=0
$$

and everything is proved. 
For every function $g(x)$ of $L_{2}\left(R^{m}\right)$ we have $g(a x) \rightarrow g(x)$ in the sense of $L_{2}\left(R^{m}\right)$ as $a \rightarrow 1$ (this is easily shown by means of an approximation of $g(x)$ by functions of $\left.C_{0}^{\infty}\left(R^{m}\right)\right)$. From this and from (2) it follows that $u((1+\epsilon) x) \rightarrow u(x)$ and $q((1+\epsilon) x) u((1+\epsilon) x) \rightarrow q(x) u(x)$ in $L_{2}\left(R^{m}\right)$ as $\epsilon \rightarrow 0$. Since $\epsilon^{-1}(q((1+\epsilon) x)-q(x))$ converges to $r q_{r}(x)$ for almost every $x$ as $\epsilon \rightarrow 0$ and is majorized by $q_{0}(x)$ (condition (II)) it follows that $\epsilon^{-1}(q((1+\epsilon) x)-q(x)) u(x) \rightarrow r q_{r}(x) u(x)$ in $L_{2}\left(R^{m}\right)$ as $\epsilon \rightarrow 0$. Consequently every term under the integral sign converges in $L\left(R^{m}\right)$; hence we may take the limit under the integral sign.

q.e.d.

3. Application to spectral theory. By means of the Virial Theorem just proved we can now show under suitable conditions on $q(x)$, that there are no eigenvalues of $H$ in certain regions of the real line; i.e. the spectral resolution of $H$ is continuous in these regions.

COROLlaRy 1. If conditions (I) and (II) are satisfied, and $q_{r}(x) \leqq 0$ for $x \in R^{m}, x \neq 0$, then $H$ does not have any eigenvalue.

Proof. Suppose $\lambda$ is an eigenvalue with the corresponding eigenfunction $u \neq 0$. Since $r q_{r}(x) \leqq 0$ we obtain from the Virial Theorem

$$
2\langle\Delta u, u\rangle=2\langle(q-\lambda) u, u\rangle \geqq\left\langle\left(2 q-2 \lambda+r q_{r}\right) u, u\right\rangle=0 .
$$

This is a contradiction since for $u \neq 0$ we have $\langle\Delta u, u\rangle<0$.

Corollary 2. Let the conditions (I), (II) and $r q_{r}(x) \leqq-\gamma q(x)$ $(0<\gamma<2, x \neq 0)$ be satisfied. Then $H$ has no eigenvalue in $[0, \infty)$.

PRoOF. (For $0<\gamma \leqq 1$ a different proof was given in [4].) Let $\lambda$ be an eigenvalue, $u(x)$ a corresponding eigenfunction; then the Virial Theorem implies $\langle((2-\gamma) q-2 \lambda) u, u\rangle \geqq 0$; hence $\langle(2-\gamma) \Delta u-\gamma \lambda u, u\rangle$ $\geqq 0$. This is possible only if $\lambda<0$.

q.e.d.

Let us now consider the Schrödinger operator of an atom or ion with a nucleus of charge $Z$ and $n$ electrons (where the nucleus has infinite mass or is supposed to be fixed); the corresponding Schrödinger operator is of the form $H$ where

$$
\begin{aligned}
q(x) & =-\sum_{j=1}^{n} \frac{Z}{r_{j}}+\sum_{1 \leq j<k} \frac{1}{r_{j k}}, \quad \text { if } r_{j}, r_{j k}>0 \\
& =0, \quad \text { if at least one of the } r_{j} \text { or } r_{j k} \text { vanishes, } \\
r_{j} & =\left\{\sum_{l=0}^{2} x_{3 j-l}^{2}\right\}^{1 / 2}, \\
r_{j k} & =\left\{\sum_{l=0}^{2}\left|x_{3 j-l}-x_{3 k-l}\right|^{2}\right\}^{1 / 2} .
\end{aligned}
$$


It is easy to show that $q(x)$ satisfies conditions (I) and (II) and is homogeneous of degree -1 . From Corollary 2 it follows that $H$ has no eigenvalue in $[0, \infty)$.

This same result holds for every $n$-particle-operator with Coulombinteractions, where the motion of the center of mass is separated out; in this case too, the potential is homogeneous of degree -1 (e.g. Weidmann [3]).

4. Remarks on Yukawa-potentials. In nuclear physics so-called Yukawa-potentials of the form

$$
p(r)=\frac{1}{r} \exp (-a r)
$$

are used frequently (for $a=0$ this reduces to Coulomb-potential). If all the potentials in an atom-like system follow such a law, then we have the Schrödinger-operator $H$ with

$$
\begin{aligned}
q(x) & =-\sum_{j=1}^{n} \frac{b_{j}}{r_{j}} \exp \left(-a_{j} r_{j}\right)+\sum_{1 \leq j<k} \frac{b_{j k}}{r_{j k}} \exp \left(-a_{j k} r_{j k}\right) \quad \text { if } r_{j}, r_{j k}>0 \\
& =0 \quad \text { if one of the } r_{j} \text { or } r_{j k} \text { vanishes. }
\end{aligned}
$$

An easy calculation yields

$$
r q_{r}(x) \leqq-q(x)+\sum_{j=1}^{n} a_{j} b_{j} .
$$

If $\lambda$ is an eigenvalue, $u(x)$ a corresponding eigenfunction of $H$, then it follows from the Virial Theorem that

$$
\begin{aligned}
0 & =\left\langle\left(2 q+r q_{r}-2 \lambda\right) u, u\right\rangle \\
& \leqq\left\langle\left(q-2 \lambda+\sum_{j=1}^{n} a_{j} b_{j}\right) u, u\right\rangle \\
& =\left\langle\left(\Delta-\lambda+\sum_{j=1}^{n} a_{j} b_{j}\right) u, u\right\rangle \\
& \left\langle\left(\sum_{j=1}^{n} a_{j} b_{j}-\lambda\right)|u|^{2} ;\right.
\end{aligned}
$$

hence $\lambda<\sum_{j=1}^{n} a_{j} b_{j}$.

For $n=1$ this means in particular that there is no eigenvalue greater than or equal to $a_{1} b_{1}$. On the other hand it is known that there is no positive eigenvalue at all (e.g. Ikebe [1], Weidmann [4]). In this case the upper bound for eigenvalues which we found here is certainly 
not optimal. For $n>1$ it is not known whether there exist positive eigenvalues or not; hence it is not known whether the bound $\sum_{j=1}^{n} a_{j} b_{j}$ is of any importance or not.

\section{REFERENCES}

1. T. Ikebe, Eigenfunction expansions associated with Schrödinger operators and their application to scattering theory, Arch. Rational Mech. Anal. 5 (1960), 1-34.

2. T. Ikebe and T. Kato, Uniqueness of the self-adjoint extensions of singular elliptic differential operators, Arch. Rational Mech. Anal. 9 (1962), 77-92.

3. J. Weidmann, On the continuous spectrum of Schrödinger operators, Comm. Pure Appl. Math. 19 (1966), 107-110.

4. ——, Zur Spektraltheorie von Sturm-Liouville-Operatoren, Math. Z. (to appear).

Universität Heidelberg, Germany 